\title{
Significância clínica de mudança em processo de psicoterapia psicodinâmica breve
}

\author{
Elisa Medici Pizão Yoshida \\ Pontifícia Universidade Católica de Campinas, Campinas-SP, Brasil
}

\begin{abstract}
Resumo: A presente investigação é parte de um trabalho em andamento cujo objetivo é a análise do processo de mudança e o resultado de psicoterapias breves em pacientes de clínicas comunitárias brasileiras. Mais especificamente, objetivou-se explorar um método para determinar a mudança clinicamente significante de sintomas avaliados por meio de medidas de auto-relato, quando dados normativos não estão disponíveis. Constitui um uso específico do bem conhecido método de Jacobson e Truax para estimar a significância clínica de um tratamento. Para calcular os escores de mudança e os pontos de corte de medidas sintomáticas utilizaram-se dados de amostra de universitários, como representativa da população funcional. Mudanças em um processo psicoterapêutico foram analisadas. Os resultados sugerem que a significância clínica da mudança pode ser determinada de forma fidedigna por meio de medidas de auto-relato. Alguns limites do método foram considerados.
\end{abstract}

Palavras-chave: Psicoterapia. Psicoterapia breve. Auto-relato. Avaliação de processos e resultados (Cuidados de Saúde).

\section{Clinical significance of change in process of brief psychodynamic therapy}

Abstract: The current investigation is part of a work in progress aimed to analyze the process of change and the outcome of brief psychotherapies in patients of Brazilian communitarian health units. Specifically, the current work aimed to explore a method to determine clinically meaningful change of symptoms evaluated by self-report measures, when normative data are not available. It consists in the specific use of the well-known Jacobson and Truax's method to determine the clinical significance of a treatment. To calculate the change scores and cutoffs of symptomatic measures, data from a sample of college students were used as representative of a functional population. Changes of one psychotherapeutic process were analyzed. Results suggest that clinical significance of change can be reliably determined by self-report measures. Some limitations of this method are considered.

Keywords: Psychoterapy. Brief psychotherapy. Self report. Outcome and process assessment (Health Care).

\section{Significación clínica del cambio en un proceso de psicoterapia psicodinámica breve}

Resumen: La presente investigación es parte de un estudio en curso que busca analizar el proceso de cambio y los resultados de psicoterapias breves de pacientes que buscan clínicas brasileñas asentada en la comunidad. Más específicamente, el presente trabajo buscó explorar un método para determinar el cambio clínicamente significante de síntomas evaluados por medio de medidas de auto relato, en situaciones en que no hay dados normativos disponibles. Constituye un empleo específico del bien conocido método de Jacobson e Truax, para estimar la significación clínica de un tratamiento. Para calcular los escores del cambio y los puntos de corte de medidas sintomáticas fueron utilizados datos de una muestra de estudiantes universitarios, como representantes de la población funcional. Cambios en un proceso terapéutico fueron analizados. Los resultados sugieren que la significación clínica del cambio puede ser determinada de manera fidedigna por medio de las medidas de auto relato. Se reflexiona sobre los límites del método.

Palabras clave: Psicoterapia. Psicoterapia breve. Auto-relato. Evaluación de procesos y resultados (Atención de Salud). 


\section{Introdução}

Este trabalho é parte de um programa de pesquisa mais amplo cujo objetivo é o estudo de mudanças obtidas por pacientes assistidos em clínicas comunitárias brasileiras, quando submetidos a psicoterapias psicodinâmicas breves (Yoshida, 2008). O projeto envolve o acompanhamento de vários processos psicoterapêuticos, utilizando um delineamento metodológico de Estudo de Caso Sistemático (ECS) (Edwards, 2007), ou ainda, Estudo de Caso Pragmático (ECP) (Eells, 2001, 2007; Messer, 2007).

O ECS é um tipo específico de pesquisa que tem como objetivo compreender os fatores que contribuem para a mudança. E para isto, avaliações de variáveis do paciente, do terapeuta e da interação entre eles, são obtidas, dentro de um contexto clínico natural. Avaliações baseadas em medidas de autorelato costumam complementar a investigação (Eells, 2001, 2007; Messer, 2007). Um dos desafios deste tipo de delineamento é a escolha do critério de mudança. Isto é, o que deve ser considerado mudança, como avaliá-la e qual "quantidade de mudança" o paciente deve apresentar para que se considere o processo bem sucedido ou não. Com o intuito de responder a estas questões, o presente estudo teve como objetivo avaliar a magnitude da mudança de um processo de psicoterapia breve, com base em medidas de auto-relato. Para tanto, foi utilizado o conceito de significância clínica da mudança (Ogles, Lambert, \& Masters, 1996). Trata-se, na realidade, de um estudo com finalidade de exploração de nova metodologia para o grupo de pesquisa, que em investigações anteriores limitou-se a avaliar a significância estatística das mudanças (Coelho Filho, 1995; Enéas, 1999; Gatti, 1994; Yoshida, 2000a; Yoshida, Gatti, Enéas, \& Coelho Filho, 1997). Neste sentido, algumas considerações de ordem metodológica são necessárias.

\section{Considerações metodológicas}

A grande diversidade das pesquisas sobre as psicoterapias na atualidade tem sido acompanhada da discussão de quais deveriam ser os objetivos das investigações e quais os delineamentos de pesquisa mais adequados. Desde os anos 1990, é crescente a preocupação em fornecer dados que sejam úteis ao clínico (Greenberg, 1994; Hill, 1994; Howard, Moras, Brill, Martinovich, \& Lutz, 1996; Seligman, 1995). Os autores argumentam que o clínico necessita de métodos válidos para avaliar sistematicamente não apenas a qualidade dos resultados possíveis para uma determinada população, mas os resultados do processo psicoterapêutico de um paciente específico e as mudanças que ocorreram ao longo dele (Eells, 2007; Messer, 2007; Ogles e cols., 1996).

Um dos desafios é o de contar com procedimentos de avaliação de mudança que detenham significância clínica e não apenas significância estatística, usualmente empregada para estimar a probabilidade de acaso envolvido na mudança ou ainda para estimar a magnitude das diferenças entre dois ou mais grupos comparados, sem contemplar as mudanças de indivíduos específicos dentro de cada grupo (Ogles e cols., 1996). O conceito de significância clínica, por outro lado, tem sido utilizado de três perspectivas diferentes: do paciente, do profissional de saúde mental (representado na maioria das vezes pelo psicoterapeuta) e da sociedade.

Do ponto de visa do paciente, a significância clínica é dada pela avaliação subjetiva que ele faz sobre a contribuição do tratamento para a sua melhora ou bem estar. Está, pois, diretamente relacionada com a satisfação em relação à terapia, ainda que as mudanças possam ter sido devidas a fatores externos a ela. Do ponto de vista do profissional, a significância clínica é dada, usualmente, com base no referencial teórico adotado por ele, e inclui diferentes níveis de inferência. Ademais, a qualidade do relacionamento estabelecido entre o paciente e o terapeuta costuma desempenhar papel relevante nesta avaliação, que pode ser beneficiada ou prejudicada, se o paciente é ou não considerado "difícil" (Messer \& Warren, 1995).

A perspectiva da sociedade é a mais diretamente relacionada com a atividade do pesquisador e é dada por, ao menos, três fatores: significância social dos objetivos do tratamento, adequação social dos procedimentos e importância social de seus efeitos (Wolf, 1978 conforme citado por Ogles e cols., 1996). Dentre estes, o mais relevante é o que considera a importância dos efeitos do tratamento. E para tanto, diferentes maneiras de avaliação foram propostas, ainda que um consenso não tenha sido atingido (Jacobson, Follete, \& Revenstorf, 1984; Jacobson \& Truax, 1991). 
Como medida dos efeitos do tratamento, o emprego da validade clínica da mudança tem se revelado como uma alternativa mais útil ao profissional, devido à sua aplicabilidade prática (Jacobson \& Truax, 1991). Uma das vantagens é que ela permite avaliar em que medida a mudança se reflete na vida do paciente de forma a aproximá-lo, por exemplo, de um nível de funcionamento compatível com o de uma pessoa "normal", ou pertencente a uma população não clínica. A título de ilustração, pode-se citar, como exemplo, a perda de peso de uma pessoa, como resultado de um tratamento específico. Ainda que uma diferença de $20 \mathrm{~kg}$. possa ser considerada estatisticamente significante, ela pode ser insuficiente para garantir uma boa qualidade de vida a alguém que, no início do tratamento, tinha $180 \mathrm{~kg}$ (Ogles e cols., 1996).

Dentre as propostas para a avaliação de significância clínica da mudança, talvez a mais conhecida seja a de Jacobson e Traux (1991). De acordo com ela, dois critérios devem ser considerados para a definição de mudança clinicamente significante: (1) o paciente deve passar de um funcionamento correspondente a uma população teoricamente disfuncional ao de uma população funcional; (2) a mudança deve ser suficientemente grande para ser atribuída a uma mudança "real" e não a erros de medida.

Considerando que o ponto de corte pode variar em função da amostra tomada como referência, uma proposta de operacionalização do primeiro critério foi feita por Jacobson e cols. (1984). Ela contempla três possibilidades: (1) após a terapia o paciente deve estar a dois desvios padrão da média da população disfuncional (na direção da funcionalidade), em relação à medida ou medidas de avaliação utilizadas; (2) após a terapia o paciente deve estar dentro da faixa de dispersão (range) de funcionamento da população normal, sendo que esta faixa é definida por dois desvios padrão da respectiva média; (3) após a terapia o paciente deve estar mais próximo da média da população funcional (normal) do que da média da população disfuncional.

Como se observa, a aplicação de qualquer uma das três alternativas depende do conhecimento das normas do teste utilizado na avaliação da mudança. Em se tratando de pesquisas brasileiras, este requisito costuma se constituir em forte obstáculo, uma vez que há um número bastante limitado de escalas ou testes de avaliação psicológica cujas normas são conhecidas. Para estas situações, Ogles e cols. (1996) aconselham que se utilize a proposta de Nietzel e Trull, 1988 (conforme citado por Ogles e cols., 1996), segundo a qual se deve adotar como ponto de corte, um desvio padrão acima da média de uma amostra considerada funcional. E neste sentido, desde que se conheça a média e o desvio padrão desta amostra, é possível estimar em que medida teria havido mudança e se esta foi suficiente para levar o paciente a atingir um funcionamento compatível com o esperado de populações não-clínicas ou funcionais.

Quanto ao segundo critério, relativo à possibilidade de mudança real, o Índice de Mudança Confiável de Jacobson e Truax (1991), ou RCI, é um dos mais utilizados, pois fornece a magnitude da diferença de medidas de avaliação feitas no pré e póstratamento, relacionando-as aos escores médios de populações funcionais e não funcionais. Para fins práticos, valores de RCI superiores a 1,96 indicam mudança confiável ou, pouco provavelmente, devida a erros de medida.

Se o paciente preenche os dois critérios, isto é, a mudança pode ser considerada confiável e o paciente encontra-se, ao final da terapia, mais próximo do funcionamento normal, diz-se que a mudança é clinicamente significante. Ambos os critérios foram empregados no presente estudo para medir a "quantidade" de mudança sintomatológica da paciente, na fase final, e também na entrevista de acompanhamento, um ano após o término de um processo de psicoterapia breve. Foram utilizadas medidas que já tinham sido objeto de investigação do grupo, em pesquisas anteriores. E nesta medida, foram escolhidas as seguintes variáveis independentes, para a avaliação do grau de mudança.

\section{Método}

\section{Participante}

Trata-se de uma mulher de 50 anos, amasiada, com ensino fundamental incompleto (cinco anos de escolaridade), dona de casa, com dez filhos (três mulheres e sete homens, sendo três falecidos). Recebia medicação antidepressiva e foi encaminhada pelo serviço de psiquiatra. 


\section{Processo psicoterapêutico}

A paciente foi atendida por um profissional do sexo masculino, de 56 anos, doutorado em Psicologia, com 35 anos de prática clínica. Foram realizadas doze sessões individuais e sessão de acompanhamento, um ano após o término. O motivo da consulta foi a falta de vontade para prosseguir cuidando de sua saúde, já que era diabética e hipertensa, e para continuar vivendo, após a morte por afogamento de um filho de 30 anos, há quatro meses, em um passeio em que estava reunida toda a família, inclusive ela. Na mesma ocasião também morreu afogado um rapaz de 14 anos, irmão do namorado de sua filha.

Estabeleceram-se como objetivos da psicoterapia, a aceitação da perda com melhora no nível da sintomatologia depressiva; recuperação do desejo de viver e de sua funcionalidade (retomada de seus afazeres domésticos); ampliação do rol de interesses e de atividades. Quanto ao desenvolvimento do processo, na fase inicial, compreendida pelas duas sessões, a paciente fez uma descrição pormenorizada do episódio do afogamento com detalhes sobre a composição familiar; referiu sonho recorrente de muita água suja, e a crença de que, quando um membro da família morre afogado, seus outros integrantes morrerão da mesma maneira. Ao mesmo tempo dizia não aceitar a idéia de destino ou "vontade de deus". Ao final do atendimento, referiu sentir-se bastante melhor, podendo falar com tranqüilidade sobre o filho, brincar com os netos, sentir-se menos impaciente e irritada, sair para compras. Admitia, todavia, que se lembrará sempre dele, especialmente em seu aniversário, Natal e datas importantes. Planejava passar o Natal na casa do filho em outra cidade, e pretendia ir à formatura da sobrinha, também, em outra cidade do interior. Referiu espontaneamente que as melhoras se deveram à psicoterapia e à possibilidade de falar sobre os acontecimentos. Na entrevista de acompanhamento (um ano), disse ter melhorado bastante e enfatizou que a melhora deveu-se ao fato de ter realizado a psicoterapia. Disse ainda que quando se sentia desanimada lembrava-se do conselho do terapeuta de que a vida deveria continuar e se sentia novamente encorajada. Nesta ocasião, afirmou que já saía às compras sozinha, participava de festas familiares, ia freqüentemente à casa do filho, cozinhava para todos.
A nora e os netos moravam com ela e seu companheiro, mas admitia que a nora deveria voltar a se casar um dia.

\section{Instrumentos}

(1) Inventário Beck de Depressão (BDI), versão brasileira de 21 itens (Cunha, 2001), desenvolvida empiricamente a partir da observação, relatos de sintomas e atitudes de pacientes psiquiátricos, não reflete qualquer teoria sobre a depressão em particular. O escore total, obtido com a soma dos escores dos itens, indica a intensidade da depressão. Pontos de corte: Mínimo (0-11); Leve (12-19); Moderado (2035); Severo (36-63). A precisão de teste-reteste para diferentes amostras brasileiras variou entre 0,40 e 0,91 (Cunha, 2001).

(2) Escala de Avaliação de Sintomas-40 EAS-40 (Laloni, 2001), trata-se de uma escala multidimensional, de auto-relato, de 40 itens, que mede sintomas psicopatológicos segundo quatro dimensões: F1-Psicoticismo (sintomas relacionados à hostilidade e idéias paranóides); F2-Obsessividadecompulsividade (sintomas relacionados a pensamentos repetitivos, seguidos de desconforto nas relações interpessoais); F3-Somatização (sintomas usualmente relacionados a distúrbios somáticos e somatoformes); F4-Ansiedade (sintomas que vão de ansiedade generalizada à ansiedade fóbica direcionada a situações e objetos específicos). Trata-se de uma adaptação para pacientes de hospitais gerais brasileiros do Symptom Checklist-90-Revised (SCL-90-R) (Derogatis, 1994), com 90 itens que avaliam nove dimensões.

Na EAS-40 a avaliação da intensidade do sintoma deve ser feita segundo três níveis: $0=$ nenhum; 1=pouco; 2=muito. O Índice Global de Severidade (GSI), um escore total, derivado tanto do número quanto da intensidade dos sintomas, é um indicador do funcionamento psicológico. A EAS-40 demonstrou boa consistência interna (de 0,73 a 0,88 ) e boa validade de construto (verificada por Análise Fatorial, Análise de Agrupamento e Análise de Correspondência) (Laloni, 2001). Evidenciou precisão para diferentes amostras brasileiras, tais como, pacientes atendidos em serviços de saúde mental, pacientes assistidos em enfermarias com diferentes doenças (Laloni, 2001) e estudantes universitários (Yoshida \& Silva, 2007), variaram entre 0,40 a 0,93 . 
(3)Versão em português da Toronto Alexithymia Scale-TAS (Yoshida, 2000b), desenvolvida por Taylor, Ryan e Bagby (1985), a escala de tipo de auto-avaliação de 26 itens, foi idealizada para medir o grau de alexitimia (uma medida da dificuldade em identificar e descrever as emoções), segundo quatro fatores: F1habilidade de identificar e descrever sentimentos e distingui-los de sensações corporais; F2-o sonhar acordado, F3-preferência por focalizar eventos externos em vez de experiências internas e F4-habilidade para comunicar os sentimentos a outras pessoas. Pauta-se em escala de tipo Likert de cinco pontos, em que (1) corresponde a discordo inteiramente, (2) discordo, (3) não sei, (4) concordo e (5) concordo plenamente. Os escores totais variam entre 26 e 130 , sendo que para escores acima de 74 (inclusive) o sujeito é considerado alexitímico e menores de 62 (inclusive) é considerado não alexitímico (Taylor e cols., 1988). Para valores intermediários, isto é, entre 63 e 73 , nada se pode afirmar. Pesquisas com diferentes amostras brasileiras sugerem que estes pontos de corte podem ser adotados em nossa realidade (Yoshida, 2006). A precisão teste-reteste para pacientes de hospital geral (Yoshida, 2007) e universitários (Yoshida $\&$ Silva, 2007) variaram entre 0,71 e 0,90 .

(4) Escala Fatorial de Ajustamento Emociona/Neuroticismo-EFN (Hutz \& Nunes, 2001), trata-se de um instrumento de auto-relato, com 82 itens, corresponde a uma medida de Neuroticismo/ Estabilidade Emocional segundo quatro facetas: vulnerabilidade, desajustamento psicossocial, instabilidade/ansiedade e depressão. Foi desenvolvida com base no modelo de personalidade dos Cinco Grandes Fatores, dos quais o Neuroticismo é uma das dimensões. De acordo com este modelo, "um alto grau de Neuroticismo identifica indivíduos propensos a sofrimento psicológico, altos níveis de ansiedade, depressão, hostilidade, vulnerabilidade, autocrítica, impulsividade, baixa auto-estima, idéias irreais, baixa tolerância à frustração e resposta de coping adaptativas" (Costa \& Widiger, conforme citado por Nunes, 2000, p.18). Escala Likert de sete pontos (de um, completamente inapropriado, "a afirmação não descreve nenhuma de minhas características”, a sete, perfeitamente apropriado, "a afirmação descreve-me perfeitamente bem”). Estimativas da precisão de pacientes de hospital geral (Yoshida, 2007) e universitários (Medeiros \& Yoshida, 2004) variaram entre 0,79e 0,93.

\section{Procedimento}

$\mathrm{O}$ atendimento, realizado nas dependências de clínica-escola, foi registrado em vídeo e áudio e as sessões transcritas. As aplicações dos instrumentos de auto-relato foram feitas por uma bolsista de iniciação científica, após a primeira entrevista, na fase medial e final do atendimento, assim como na entrevista de acompanhamento. A aplicação de todos os instrumentos foi feita de maneira assistida, com a leitura dos itens e das alternativas de resposta, realizada pela bolsista. Em face dos objetivos deste trabalho, aqui não são apresentados os resultados da avaliação medial. O projeto foi aprovado pelo Comitê de Ética em Pesquisa da instituição.

\section{Critérios de mudança}

Adotou-se como critérios de mudança os índices propostos por Jacobson e Truax (1991), que fornecem indicações da significância clínica da mudança. Conforme referido, de acordo com eles, a mudança observada detém significância clínica se: (1) o paciente passar de um funcionamento correspondente a uma população teoricamente disfuncional para o de uma população funcional, e (2) a mudança foi suficientemente grande para ser atribuída a uma mudança "real" e não a erros de medida.

Seguindo a sugestão de Nietzel e Trull (1998 conforme citado por Ogles e cols., 1996), para calcular os pontos de corte de cada instrumento e com isto estabelecer a faixa de funcionalidade, definiu-se o valor de um desvio padrão acima da média de uma amostra de estudantes universitários. E para a estimativa da magnitude de mudança confiável, calculou-se a mudança mínima necessária entre os escores do teste no início e ao término do atendimento para um RCI=1,96 (Jacobson \& Truax). Para tanto, foi empregada a fórmula de Jacobson e cols. (1984) para a avaliação de resultados de psicoterapia e aperfeiçoada por Christensen e Mendoza (1986 conforme citado por Jacobson \& Truax), de acordo com a qual, o RCI corresponde à razão da diferença entre os escores pós-teste $\left(\mathrm{x}_{2}\right)$ e teste $\left(\mathrm{x}_{1}\right)$ e o erro padrão da diferença entre os dois escores do teste $\left(\mathrm{S}_{\text {diff }}\right)$. Isto, é: $\mathrm{RCI}=\mathrm{x}_{2}-\mathrm{x}_{1}, \mathrm{~S}_{\text {diff }}$.

$\mathrm{O} \mathrm{S}_{\text {diff }}$ corresponde à raiz quadrada do dobro do erro padrão de medida $\left(\mathrm{S}_{\mathrm{E}}\right)$, ao quadrado. Isto é, 
$S_{\text {diff }}=\sqrt{2\left(S_{E}\right)^{2} \cdot E}$, o erro padrão de medida é igual ao produto do desvio padrão da amostra $\left(\mathrm{s}_{1}\right)$ e a raiz quadrada de 1 menos o coeficiente de precisão testereteste $\left(r_{x x}\right)$ da medida em questão $\left(S_{E}=s_{1} \sqrt{1-r_{x x}}\right)$. Ou seja, para a estimativa do RCI é necessário ter o valor da precisão de teste-reteste e do desvio-padrão relativos a uma determinada amostra.

Conforme referido, apesar de haver normas brasileiras para o BDI (Cunha, 2001), foram calculados, para o presente estudo, tanto o ponto de corte, quanto o valor de escore de mudança correspondente a um RCI igual a 1,96, para uma amostra de estudantes universitários. Tomou-se como referência, o coeficiente de precisão de teste e reteste, a média e o desvio padrão de uma amostra de 1784 estudantes, reportados por Cunha (Tabela 1). Em relação aos demais instrumentos de avaliação, foram utilizados estudos com amostras de estudantes universitários (Medeiros \& Yoshida, 2004; Yoshida, 2000b; Yoshida $\&$ Silva, 2007), cujos valores, correspondentes a cada um dos critérios, encontram-se dispostos na Tabela 1. A opção por amostras de universitários foi baseada no fato de que este era o único tipo de amostra não-clínica, para o qual se dispunha de ao menos um estudo de precisão de teste e reteste, valores de escores médios e desvios padrão, para todos os instrumentos empregados na pesquisa.

Tabela 1

Média, Desvio Padrão, Precisão, Escores de mudança e Pontos de corte do BDI, TAS, EAS-40 e EFN com base em amostras de universitários

\begin{tabular}{lccccc}
\hline \multicolumn{1}{c}{ Instrumento } & Média & Desvio Padrão & Precisão & Mudança $^{1}$ & Ponto de Corte $^{2}$ \\
\hline BDI $(\mathrm{n}=1784)^{3}$ & 6,47 & 5,80 & 0,40 & 12 & 12 \\
TAS $(\mathrm{n}=581)^{4}$ & 63,13 & 10,12 & 0,71 & 15 & 73 \\
EAS-40 $(\mathrm{n}=113)^{5}$ & 0,60 & 0,41 & 0,93 & 0,30 & 1 \\
EAS-40 F1 & 0,59 & 0,42 & 0,80 & 0,52 & 1 \\
EAS-40 F2 & 0,76 & 0,40 & 0,84 & 0,44 & 1 \\
EAS-40 F3 & 0,55 & 0,42 & 0,87 & 0,42 & 1 \\
EAS-40 F4 & 0,49 & 0,41 & 0,91 & 0,34 & 1 \\
EFN 1 (n=60) ${ }^{6}$ & 64,01 & 21,65 & 0,86 & 22 & 36 \\
EFN2 & 22,56 & 9,25 & 0,93 & 7 & 90 \\
EFN3 & 69,76 & 20,73 & 0,87 & 21 & 52 \\
EFN4 & 38,1 & 13,82 & 0,87 & 14 & 50 \\
\hline
\end{tabular}

1. Escore de mudança necessária entre o pré e pós para um $\mathrm{RCI}=1,96$ usando um desvio padrão

e a precisão para uma dada amostra de universitários.

2. Ponto de corte: representa 1 desvio padrão acima da média do grupo funcional (universitários).

3. Cunha (2001).

4. Yoshida (2000b).

5. Yoshida e Silva (2007).

6. Medeiros e Yoshida (2004).

Com base na diferença entre os escores inicial e final, e, inicial e do acompanhamento, de cada instrumento, foi possível considerar se teria havido ou não "melhora" (mudança superior a um RCI>1,96). Tomando como base o valor do ponto de corte, foi possível estabelecer se a paciente teria se "recuperado". Isto é, se ela teria atingido um nível compatível com o observado na população funcional, no caso, representada por estudantes universitários.

\section{Resultados}

Os resultados das avaliações realizadas com os instrumentos de auto-relato nas sessões inicial, final e de acompanhamento (um ano após o término), estão apresentados na Tabela 2. Ela indica ainda, se a diferença entre o escore inicial e final e entre o inicial e o do acompanhamento, de cada instrumento de avaliação, descontada a possibilidade de erro da medida, foi suficientemente grande para se falar em 
mudança $(\mathrm{S})$ ou não $(\mathrm{N})$. Em caso afirmativo, indicou-se então que houve "melhora". A Tabela 2 informa ainda, nas duas últimas colunas, se a paciente atingiu (S), ou não $(\mathrm{N})$, um nível funcional na medida em questão. Isto é, se seu desempenho foi equivalente ao esperado para sujeitos pertencen- tes a estratos não-clínicos da população, o que sinaliza se houve ou não "recuperação". Resultados positivos de "melhora" e de "recuperação" apontam, portanto, para um nível de mudança que detém significância clínica, de acordo com os critérios adotados.

Tabela 2

Escores das sessões inicial e final e de follow-up e status de mudança no BDI, TAS, EAS-40 e EFN $(n=1)$

\begin{tabular}{lccccccc}
\hline \multicolumn{1}{c}{ Escala } & Inicial & Final & Melhorou $^{1}$ & $\begin{array}{c}\text { Sessão } \\
\text { Recuperou-se }^{2}\end{array}$ & Follow-up & Melhorou $^{1}$ & Recuperou-se $^{2}$ \\
\hline BDI & 37 & 24 & $\mathrm{~S}$ & $\mathrm{~N}$ & 22 & $\mathrm{~S}$ & $\mathrm{~N}$ \\
TAS - T & 73 & 49 & $\mathrm{~S}$ & $\mathrm{~S}$ & 63 & $\mathrm{~N}$ & $\mathrm{~S}$ \\
EAS-40 T & 1,68 & 1,28 & $\mathrm{~S}$ & $\mathrm{~N}$ & 0,83 & $\mathrm{~S}$ & $\mathrm{~S}$ \\
EAS-40 F1 & 1,50 & 0,90 & $\mathrm{~S}$ & $\mathrm{~S}$ & 0,60 & $\mathrm{~S}$ & $\mathrm{~S}$ \\
EAS-40 F2 & 1,90 & 1,50 & $\mathrm{~N}$ & $\mathrm{~N}$ & 1,20 & $\mathrm{~S}$ & $\mathrm{~N}$ \\
EAS-40 F3 & 1,60 & 1,70 & $\mathrm{~N}$ & $\mathrm{~N}$ & 1,10 & $\mathrm{~S}$ & $\mathrm{~N}$ \\
EAS-40 F4 & 1,70 & 1,00 & $\mathrm{~S}$ & $\mathrm{~S}$ & 0,40 & $\mathrm{~S}$ & $\mathrm{~S}$ \\
EFN1 & 107 & 113 & $\mathrm{~N}$ & $\mathrm{~N}$ & 80 & $\mathrm{~S}$ & $\mathrm{~S}$ \\
EFN2 & 18 & 14 & $\mathrm{~N}$ & - & 16 & $\mathrm{~N}$ & - \\
EFN3 & 117 & 106 & $\mathrm{~N}$ & $\mathrm{~N}$ & 101 & $\mathrm{~N}$ & $\mathrm{~N}$ \\
EFN4 & 87 & 86 & $\mathrm{~N}$ & $\mathrm{~N}$ & 75 & $\mathrm{~N}$ & $\mathrm{~N}$ \\
\hline
\end{tabular}

${ }^{1} \mathrm{RCI}>1,96$;

${ }^{2}$ Dentro da faixa de variação funcional.

Em relação ao BDI, a queda nos escores, ao longo do tempo (Tabela 2), sinaliza que teria havido um abrandamento da sintomatologia em relação ao início do processo terapêutico. Observa-se que entre a primeira avaliação (37 pontos) e a da fase final da psicoterapia (24 pontos) houve uma diferença de 13 pontos. $\mathrm{E}$, em relação à realizada na entrevista de acompanhamento (22 pontos), a diferença foi de 15 pontos. Nos dois casos, portanto, a diferença foi superior aos 12 pontos (Tabela 1) necessários para se considerar que a mudança teria sido real e não devida a um erro de mensuração (Jacobson \& Traux, 1991). Todavia, apesar da melhora, a paciente seguia com nível moderado de depressão, um ano após o término do atendimento (22 pontos), não se podendo portanto falar em recuperação.

Quanto à medida de alexitimia, fornecida pela TAS, o escore da sessão inicial (73) (Tabela 2), correspondia ao ponto de corte indicativo de alexitimia (Tabela 1). Ao final do processo, a paciente evoluiu para o nível não-clínico (49 pontos) e com uma diferença de 24 pontos de escore, o que permite falar em mudança clinicamente significante. No acompanhamento, no entanto, o escore de 63 pontos (Tabela 2) indicava que ela não mantivera a melhora em relação ao início do processo, tendo a diferença entre as duas avaliações caído para apenas 10 pontos. Neste caso, não se pode garantir que a diferença estivesse realmente indicando uma mudança ou, se tratava-se apenas de variação devida a erro de medida. Com base no critério de mudança adotado, não se pode, portanto, garantir que a paciente tenha realmente melhorado em relação à capacidade de expressão dos afetos, apesar de, ao final, o escore da escala já se encontrar dentro da faixa de normalidade.

No que concerne aos sintomas psicopatológicos, conforme avaliados pela EAS-40, ao final do aten- 
dimento a paciente apresentava melhora mas ainda mantinha nível sintomatológico compatível com o de população clínica. No entanto, no acompanhamento já teria havido mudança clinicamente significante na gravidade da sintomatologia geral (EAS-40T) (diferença entre as duas mensurações superior a 0,30 e escore inferior a 1, conforme Tabela 1). Este resultado teria sido mais fortemente determinado pelas mudanças nos sintomas congregados nas dimensões psicoticismo (F1) e ansiedade (F4) que registraram mudanças mais expressivas. Em relação aos sintomas das dimensões obsessividade-compulsividade (F2) e somatização (F3) foram observadas melhoras, sem todavia atingir um nível não-clínico de funcionamento.

Em relação às medidas de neuroticismo, não foram identificadas mudanças ao final do atendimento em nenhuma dos quatro componentes avaliado. Apenas no acompanhamento foi identificada mudança clinicamente significante no nível de vulnerabilidade (EFN1) (Tabela 2). Efetivamente, a paciente obteve mais de 22 pontos de escore entre uma medida e outra e escore final inferior a 86 (Tabela 1). No componente desajustamento psicossocial (EFN2), desde o início, a paciente apresentou nível compatível com o de população não-clínica (<32) (Tabela 1), o que se manteve ao longo do processo, até a entrevista de acompanhamento. Trata-se de resultado esperado, uma vez que os itens deste fator medem comportamentos encontrados em pessoas com transtornos graves de personalidade e funcionamento borderline, o que não correspondia ao perfil psicopatológico da paciente.

Quanto aos demais componentes do neuroticismo, ansiedade (EFN3) e depressão (EFN4), os índices se mantiveram muito elevados até a entrevista de acompanhamento, sendo, respectivamente, 101 e 75 (Tabela 2). Portanto, muito acima dos pontos de corte apresentados à Tabela 1 (90 e 52). No que concerne à magnitude da mudança do componente que mede ansiedade (EFN3), a diferença foi, respectivamente, de apenas 11 e 16 pontos de escores entre a primeira medida e as do final e do acompanhamento (Tabela 2). E, portanto, bem inferiores aos 21 pontos necessários (Tabela 1). No componente que mede depressão (EFN4), as diferenças foram de apenas 1 e 12 pontos (Tabela 2), quando seria necessária diferença igual ou superior a 14 pontos (Tabela 1). Ou seja, de acordo com os resultados, não é possível falar que a paciente teria apresentado mudança clinicamente significante nas facetas ansiedade e depressão do neuroticismo, mantendo-se dentro de patamares clínicos em ambas, tanto ao final quanto um ano após o término da psicoterapia.

\section{Discussão}

O emprego do expediente proposto por Nietzel e Trull, 1998 (conforme citado por Ogles e cols., 1996), de utilizar medidas provenientes de amostras não-clínicas para a definição de mudança clinicamente significante, quando do emprego dos critérios de Jacobson e Truax (1991), mostrou-se exequiível e bastante útil. Como referido, ele deve ser empregado em situações em que as normas do teste não são conhecidas.

No caso brasileiro, em que só recentemente tem havido um movimento mais consistente de validação de testes e escalas, a ausência de normas que permitam situar a pessoa em relação à população (funcional e disfuncional) mais ampla, segue sendo um obstáculo a ser superado para a maioria dos instrumentos. Dentre os utilizados no presente estudo, apenas o BDI conta com normas brasileiras. E, como se viu, o emprego dos critérios de Nietzel e Trull, 1998 (conforme citado por Ogles e cols., 1996) aos valores da média e desvio padrão de apenas uma amostra de estudantes (Cunha, 2001), permitiu chegar a um ponto de corte compatível com o limiar inferior de sintomas depressivos de intensidade leve e, ao mesmo valor do ponto de corte relatado no manual do teste por Cunha (2001).

No que respeita ao critério de confiança na mudança, tomando como base um RCI igual a 1,96, a diferença segura deveria ser de 12 pontos de escore para o BDI (Tabela 1). Este valor está muito próximo do valor de 13 pontos, encontrado por Ogles e cols. (1996), com base na estimativa mais conservadora da precisão de teste e reteste $(r=0,48)$ e da maior variância estimada, de uma amostra não-deprimida da população geral norte-americana $(\sigma=6,47)$. Aliás, cabe destacar que aqueles autores sugerem que uma diferença de nove pontos e escores inferiores a 13 pontos parecem ser critérios realistas para indicar que a pessoa atingiu um funcionamento compatível com o de amostra funcional ou não-clínica. 
Em relação à TAS, o ponto de corte encontrado foi 73 , quando na literatura internacional tem se empregado 74 (Taylor e cols., 1988). Ou seja, também em relação à medida de alexitimia, o ponto de corte utilizado parece ser bastante confiável. Quanto à diferença de 15 pontos entre o pré e pós-teste também parece ser realista, considerando que no final da psicoterapia, de apenas 11 sessões, a paciente foi capaz de obter uma mudança bastante acentuada em relação ao início da terapia ( 24 pontos). Estes dados estariam de fato corroborando uma mudança que pode ser observada clinicamente. Tanto no registro em vídeo quanto em áudio das sessões, é possível observar que a paciente passou a verbalizar de forma muito mais evidente suas emoções e sentimentos, o que teria certamente se refletido na medida de alexitimia. Esta habilidade teria, todavia, sofrido um decréscimo por ocasião do acompanhamento, em que se registrou uma diferença de apenas 10 pontos de escore na TAS, quando comparada ao início do atendimento. Acredita-se que a falta de acompanhamento psicoterápico tenha contribuído para este recuo. Efetivamente, o psicoterapeuta teve um papel relevante no estímulo à expressão das emoções pela paciente, ao adotar uma conduta empática e suportiva frente seus sentimentos. Terminada a psicoterapia, a paciente parece não ter encontrado no seu ambiente familiar, suporte suficiente para continuar expressando suas emoções. De toda forma, é preciso assinalar a utilidade do critério adotado para a estimativa da mudança, posto que permitiu inclusive identificar uma possível regressão na evolução da paciente.

No que respeita às avaliações realizadas com a EAS-40, também foi possível acompanhar a evolução da mudança quanto à severidade da sintomatologia, nas quatro dimensões avaliadas. No momento do acompanhamento, a paciente estava muito melhor em todas elas, apesar de não ter atingido a faixa de funcionalidade nas dimensões, obsessividade-compulsividade (F2) e somatização (F3).

Os sintomas da dimensão obsessividadecompulsividade, conforme referido, estão relacionados a pensamentos repetitivos, seguidos de desconforto nas relações interpessoais. No caso da paciente, estavam ligados à dificuldade que enfrenta- va para superar a dor da morte traumática do filho, à qual presenciara sem poder fazer nada, e à percepção de que sua família não lhe dava o suporte necessário para expressar seu sofrimento (queixava-se de que os outros filhos não sentiam e não respeitavam a dor que ela sentia). Dito de outra forma, a paciente apresentava dificuldade de reconhecer em sua rede de pessoas significativas (Moré, 2005), o apoio e o conforto necessários para superar seu sofrimento.

Uma das características de situações de stress intenso, e que podem ser genericamente chamadas de síndrome de resposta ao stress, corresponde a uma "inundação" por emoções violentas e fortes que acompanham sintomas intrusivos, desencadeados pela recordação das imagens da situação estressora (Horowitz, 2005). Em relação à somatização, caracterizada por sintomas usualmente relacionados a distúrbios somáticos e somatoformes, aparecia relacionada ao pouco ânimo que tinha para se dedicar às tarefas de seu dia-a-dia, dentro deste contexto geral de dificuldade de superar a perda sofrida. $\mathrm{Ou}$ seja, a maior resistência para mudança nas dimensões obsessividade-compulsividade e somatoforme, estaria em conformidade com a gravidade da situação de vida enfrentada pela paciente, sendo, portanto, compreensível que ainda não registrassem níveis compatíveis com os da população funcional.

Quanto às medidas de neuroticismo, foi observada mudança clinicamente significante apenas no primeiro componente da EFN, relativo o grau de vulnerabilidade. E mesmo assim, apenas por ocasião da entrevista de acompanhamento. Ao final do atendimento, em nenhum componente foram registradas melhora e/ou recuperação. A este respeito, cabe mencionar, que o neuroticismo é entendido como um traço de personalidade e como tal tende a ser mais resistente a mudanças. Especialmente quando se tratam de psicoterapias breves, mudanças significantes na severidade das dimensões que integram o neuroticismo, mais raramente observadas. A observação, aparentemente paradoxal, de que a paciente teria apresentado mudança clinicamente significante quanto aos sintomas de ansiedade, de acordo com a EAS-40, mas não, de acordo com a EFN, pode ser explicada pelo fato de que elas avaliam aspectos distintos das manifestações de ansiedade. Enquanto a 
avaliação da EAS-40 é estritamente sintomatológica, a da EFN revela uma das características do traço de personalidade, o neuroticismo.

\section{Considerações finais}

Os resultados do estudo permitem concluir que: (1) o emprego dos critérios de avaliação de mudança clinicamente significante, propostos por Jacobson e Truax (1991), podem ser empregados, mesmo quando não se dispõe de normas para as medidas de avaliação; (2) é possível estimar-se os índices necessários para a aplicação dos critérios de Jacobson e Truax (1991), desde que sejam conhecidos, a média, desvio padrão e índice de precisão de um instrumento, para uma amostra representativa de um estrato da população; (3) um desvio padrão acima do escore médio de uma amostra considerada funcional (Nietzel \& Trull, 1988 conforme citado por Ogles e cols., 1996), somado à estimativa da magnitude necessária de mudança, para um RCI maior do que 1,96 (Jacobson \& Truax, 1991), relativa a esta mesma amostra, mostrou-se útil para a determinação da significância clínica da mudança, avaliada por meio de instrumentos de auto-relato; (4) foi possível situar a paciente em relação a cada medida, tomando como referência uma distribuição de uma amostra de estudantes (considerada funcional) (Tabela 2); (5) a diferença de nível educacional existente entre a paciente (cinco anos de escolaridade) e as amostras de universitário parece não ter comprometido os resultados, visto que os pontos de corte e da magnitude das diferenças ficaram muito próximos dos de estudos estrangeiros e mesmo de estudos brasileiros, como no caso do BDI e da TAS; (6) os pontos de corte e a magnitude necessária das mudanças utilizados podem ser aplicados a outros processos psicoterapêuticos; (7) a aplicação destes índices permite estimar a eficiência de processos psicoterapêuticos de diferentes abordagens teóricas e, eventualmente, compará-los entre si; (8) é possível comparar o desempenho de diferentes pacientes atendidos por um único terapeuta e também, um mesmo paciente, submetido a diferentes processos terapêuticos, independentemente de quem seja o terapeuta.

Apesar destas possibilidades, e de outras tantas que se possa aventar, é necessário ter-se em conta que estes índices estão baseados no comportamento de uma amostra específica, que pode não ser totalmente adequada para balizar o julgamento de um paciente específico. Dentre as limitações cabe destacar as relacionadas às variações da dispersão, da assimetria e/ou da curtose (achatamento) da curva de distribuição da variável, o que pode elevar excessivamente, ou ao contrário, tornar muito baixo o valor do ponto de corte, que delimitará o nível de funcionalidade ou de disfuncionalidade. Além disto, outras características, tais como, o perfil sócio-demográfico, podem também tornar irreal os critérios para comparação de um determinado paciente com a amostra representativa da população funcional.

Finalmente, cabe mencionar a questão da intersecção entre as curvas de distribuição das populações funcionais e disfuncionais. Nos casos em que há um alto grau de intersecção, um desvio padrão acima da média de uma amostra funcional pode ser um critério muito brando de mudança. Quando, ao contrário, as duas curvas são claramente distintas, o critério pode se tornar excessivamente exigente. Em cada caso, portanto, cabe ao pesquisador, ou ao clínico, avaliar criticamente a representatividade da amostra tomada como parâmetro de comparação e decidir quanto à utilidade prática de seu emprego. Apesar dos limites dos critérios utilizados, acredita-se que eles ofereçam suficientes vantagens para o seu emprego em pesquisa de avaliação de mudança em psicoterapias breves, e que possam, eventualmente, vir a ser utilizados por outros pesquisadores brasileiros, que queiram contar com uma forma objetiva e confiável de avaliação de mudança, sem desconsiderar a relevância clínica do procedimento.

\section{Referências}

Coelho Filho, J. G. (1995). Processo e aliança terapêutica de pacientes borderline em psicoterapia breve psicodinâmica. Dissertação de mestrado não-publicada, Pontifícia Universidade Católica de Campinas, Campinas, SP.

Cunha, J. A. (2001). Manual da versão em português das Escalas Beck. São Paulo: Casa do Psicólogo.

Derogatis, L. R. (1994). Symptom Checklist-90-R (SCL-90-R). Administration, scoring, and procedures manual (3rd. ed.). Mineapolis, US: National Computer Systems. 
Edwards, D. J. A. (2007). Collaborative versus adversarial stances in scientific discourse: Implications for the role of systematic case studies in the development of evidence-based practice in psychotherapy [Versão eletrônica]. Pragmatic Case Studies in Psychotherapy, 3(1), 6-34. Recuperado em 08 fevereiro 2008, de http:// pcsp.libraries. rutgers.edu.

Eells, T. D. (2001). Update on psychotherapy case formulation research. Journal of Psychotherapy: Practice \& Research, 10, 277-281.

Eells, T. D. (2007). Generating and generalizing knowledge about psychotherapy from pragmatic case studies [Versão eletrônica]. Pragmatic Case Studies in Psychotherapy, 3(1), 35-54. Recuperado em 08 fevereiro 2008, de http://pcsp.libraries. rutgers.edu.

Enéas, M. L. E. (1999). Exploração de processos de psicoterapias por meio da Escala Rutgers de Progresso em Psicoterapia. Tese de doutorado não-publicada, Pontifícia Universidade Católica de Campinas, Campinas, SP.

Gatti, A. L. (1994). Alterações no Rorschach após psicoterapia breve psicodinâmica. Dissertação de mestrado não-publicada, Pontifícia Universidade Católica de Campinas, Campinas, SP.

Greenberg, L. S. (1994). The investigation of change: Its measurement and explanation. In R. L. Russell (Ed.), Reassessing psychotherapy research (pp. 114-143). New York: Guilford Press.

Hill, C. E. (1994). From an experimental to an exploratory naturalistic approach to studying psychotherapy process. In R. L. Russell (Ed.), Reassessing psychotherapy research (pp. 144165). New York: Guilford Press.

Horowitz, M. J. (2005). Treatment of stress response syndromes. Washington, DC: American Psychiatric Publishing.

Howard, K., Moras, K., Brill, P. Martinovich, Z., \& Lutz, W. (1996). Evaluation of psychotherapy: Efficacy, effectiveness, and patient progress. American Psychologist, 51, 1025-1030.

Hutz, C. S., \& Nunes, C. H. S. S. (2001). Escala Fatorial de Ajustamento Emocional/Neuroticismo. São Paulo: Casa do Psicólogo.
Jacobson, N. S., Follette, W. C., \& Revenstorf, D. (1984). Psychotherapy outcome research: Methods for reporting variability and evaluating clinical significance. Behavior Therapy, 15, 336352.

Jacobson, N. S., \& Truax, A. P. (1991). Clinical significance: A statistical approach to defining meaningful change in psychotherapy research. In A. E. Kazdin (Ed.), Methodological issues and strategies in clinical research (2th ed., pp. 521538). Washington, DC: American Psychological Association.

Laloni, D. T. (2001). Escala de avaliação de sintomas-90-R SCL-90-R: Adaptação, precisão e validade. Tese de doutorado não-publicada, Pontifícia Universidade Católica de Campinas, Campinas, SP.

Medeiros, F. S., \& Yoshida, E. M. P. (2004). Validade simultânea da Escala Fatorial de Ajustamento Emocional/Neuroticismo-EFN com estudantes universitários [Resumo]. Encontro de Iniciação Científica, 9, 101.

Messer, S. B. (2007). Psychoanalytic case studies and the pragmatic case study method [Versão eletrônica]. Pragmatic Case Studies in Psychotherapy, 3(1), 55-58. Recuperado em 08 fevereiro 2008, de http://pcsp.libraries.rutgers.edu.

Messer, S. B., \& Warren, C. S. (1995). Models of brief psychotherapy: A comparative approach. New York: Guilford Press.

Moré, C. O. O. (2005). As redes pessoais significativas como instrumento de intervenção psicológica no contexto comunitário [Versão eletrônica]. Paidéia (Ribeirão Preto), 15, 287-297.

Nunes, C. H. S. S. (2000). A construção de um instrumento de medida para o fator neuroticismo/ estabilidade emocional dentro do Modelo de Personalidade dos Cinco Grandes Fatores. Dissertação de mestrado não-publicada, Universidade Federal do Rio Grande do Sul, Porto Alegre. Recuperado em 23 março 2004, de www.psicologia.ufrgs.br/laboratório.

Ogles, B. M., Lambert, M. J., \& Masters, K. S. (1996). Assessing outcome in clinical practice. Boston: Simon \& Schuster. 
Seligman, M. E. P. (1995). The effectiveness of psychotherapy: The consumer reports study. American Psychologist, 50, 965-974.

Taylor, G. J., Bagby, R. M., Ryan, D. P. Parker, J. D., Doody, K. F., \& Keefe, P. (1988). Criterion validity of the Toronto Alexithymia Scale. Psychosomatic Medicine, 50, 500-509.

Taylor, G. J., Ryan, D., \& Bagby, R. M. (1985). Toward the development of a new self-report alexithymia scale. Psychotherapy and Psychosomatics, 44, 191-199.

Yoshida, E. M. P. (2000a). Mudança em psicoterapia psicodinâmica breve: Eficácia adaptativa e funcionamento defensivo. Revista Brasileira de Psicoterapia, 2, 261-276.

Yoshida, E. M. P. (2000b). Toronto Alexthymia ScaleTAS: Precisão e validade da versão em português. Psicologia: Teoria e Prática, 2(1), 59-74.

Yoshida, E. M. P. (2006). Validade da versão em português da Toronto Alexithymia Scale. Avaliação Psicológica, 5, 235- 238.

Yoshida, E. M. P. (2007). Validade da versão em português da Toronto Alexithymia Scale-TAS em amostra clínica. Psicologia: Reflexão e Crítica, 20,389-396.

Yoshida, E. M. P. (2008). Mudança em psicoterapias breves: Características de personalidade, conflito interpessoal e estratégia terapêutica. Relatório de pesquisa não-publicado, Pontifícia Universidade Católica de Campinas, Campinas, SP.

Yoshida, E. M. P., Gatti, A. L., Enéas, M. L. E., \& Coelho Filho, J. G. (1997). Aliança terapêutica, transferência e motivação num processo de psicoterapia breve. Mudanças: Vértices de Observação, 7, 141- 154.

Yoshida, E. M. P., \& Silva, F. R. C. S. (2007). Escala de Avaliação de Sintomas-40 (EAS-40): Validade e precisão em amostra não clínica. Psicologia Escolar e Educacional, 11, 89-99.
Artigo recebido em 22/10/2007.

Aceito para publicação em 25/07/2008.

A autora agradece à colaboração do colega Sebastião Elyseu Júnior, que realizou a psicoterapia e à bolsista de iniciação científica (FAPESP) Elisa Frederich Penteado, que auxiliou na coleta dos dados. Este trabalho foi originalmente apresentado na conferência de encerramento do $14^{\circ}$ Encontro Serviços-Escola de Psicologia do Estado de São Paulo Saberes e Fazeres: Os caminhos da integração, realizado de 5 a 7 de outubro de 2006, na Universidade Presbiteriana Mackenzie, São Paulo.

Endereço para correspondência:

Profa. Dra. Elisa Medici Pizão Yoshida. Pontifícia Universidade Católica de Campinas (Campus II). Programa de Pós Graduação em Psicologia (Prédio Administrativo). Av. John Boyd Dunlop, s/nº Jd. Ipaussurama. CEP 13060-904. Campinas, SP. E-mail: eyoshida.tln@terra.com.br

Elisa Medici Pizão Yoshida é Professor Doutor da Pontifícia Universidade Católica de Campinas-SP. 